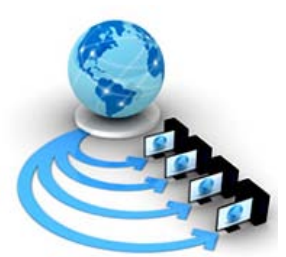

Volume 8, No. 9,November-December 2017

International Journal of Advanced Research in Computer Science

RESEARCH PAPER

Available Online at www.ijarcs.info

\title{
A LOCAL APPROACH TO GABOR WAVELET BASED FACE RECOGNITION SYSTEM
}

\author{
Kailash S. Sharma \& Ramchandra R. Manthalkar \\ Electronics \& Telecommunication Engineering Deptt. \\ SGGSIET, \\ Vishnupuri, Nanded, India
}

\begin{abstract}
Gabor wavelet has proved to be an effective tool in extracting important features from the face images. In this research work we are proposing a face recognition system which uses Gabor filter bank to create Gabor feature images. The Gabor feature images with their different orientation and scale has increased the feature dimensionality. To reduce these features and form the final feature descriptor a local approach based on the regional histogram formation is used. In this approach, the featured images are divided into different regions. The histogram of each region is calculated and concatenated to form the final feature descriptor. Chi square similarity measure is used for classification. The effectiveness of the algorithm is justified on the face images which have illumination variations and pose variations in it.
\end{abstract}

Keywords:Gabor wavelet; histogram; down sampling; feature vector; face descriptor; similarity measure

\section{INTRODUCTION}

Human face has very complex structure. The presence of various components like eyes, eye brows, nose, lips etc. adds complexity in the automatic recognition of faces. All these components have certain fixed shape, size and their proportion in the total face area. Even though it has a complex structure, it has a definite shape and can be detected very easily. But when it comes to discriminate between the two faces, the act which can be done by human sensory system within a fraction of second and without making any special arrangement, may take a series of well-defined actions to be performed by an automatic computer system [1].

In this work a face recognition system based on the local feature formation using Gabor wavelet is proposed. As Gabor wavelets works similar to human cortex system, it will be able to extract the features of the face images which may discriminate between two face images correctly. Human face with particular type of non-repetitive texture contains multiple curves over it, which may have different frequency contents present within. To extract such features Gabor wavelet with multiple filters (filter bank) are used[2][3].

The contribution made by this work can be summarised as: 1) A detailed analysis of human face from the point of view of extracting features from different face regions. 2) The features obtained by filtering with Gabor wavelets are transformed into face feature descriptor using local histogram forming approach.

The rest of the work is organized as follows. In section II we review the related work, in section III we introduce and explain our proposed system. Experiments performed \& results are taken in Section IV. Finally we conclude alongwith the future scope in section $\mathrm{V}$.

\section{LITERATURE SURVEY}

In face recognition two approaches, global and local are commonly used. In global approaches the features extracted from entire face image are considered for classification. The popular methods using global approaches are based on subspace analysis using principle component analysis (PCA)[4], linear discriminant analysis (LDA) [5] and independent component analysis (ICA) [6].Whereas in local approaches the features are obtained by considering the pixels in the neighborhood. These can be further divided based on the way the local features are extracted.

The first approach is to divide the image into different rectangular regions. Process each region separately and determine the histogram by taking ' $n$ ' number of bins. Where ' $n$ ' can be varied from 1 to number of gray levels in the image. Here the histogram of each region is representing the local features for that region. All such local features are finally concatenated together to represent the face by a feature descriptor. Finally a similarity measure like Chi Square, Mahalobanis distance, Cosine, or Euclidean distance is used for the classification. This approach is widely followed in local binary pattern (LBP)[7], local ternary pattern (LTP)[8], local derivative pattern (LDP)[9], local directional number pattern (LDN)[10], local vector pattern (LVP)[11] etc.

In second approach based on the fiducial points a grid is formed. At each fiducial point Gabor features with different resolutions are extracted. Such features are finally combined together to form the face feature descriptor. Mahalobanis or cosine distance mesures are used for classification. The main challenge in this approach is locating automatically the fiducial points prior to extract the features. This approach is used in dynamic link architecture (DLA)[12], elastic bunch graph matching (EBGM)[13] etc.

In third approach, number of strongest feature points on the face are located first and then the features are extracted around that point. These features at different points are concatenated together to form the feature descriptor. This proved to be an effective approach but if some of the points are missed out, it will result in miss-classification. For the 
detail explanation of different face recognition system one can refer the survey made by [3][14][15].

Though the Gabor filtered feature images are robust, they are computationally intensive. The dimensionality of this approach is directly increased by the number of times, the scale and orientation of the Gabor wavelets are used. In the proposed approach our main intention is to have features which can discriminate between the two face images, there is no need to represent back that image. Hence we are considering only two scales and eight rotations. The features are further reduced using down sampling and reducing the number of histogram bins used in the local processing of the image, before finding the final face feature descriptor.

\section{SYSTEM METHODOLOGY}

In the proposed system, first we are forming a code book using the gallery images and then the code of probe image is compared with the code of each and every gallery image in

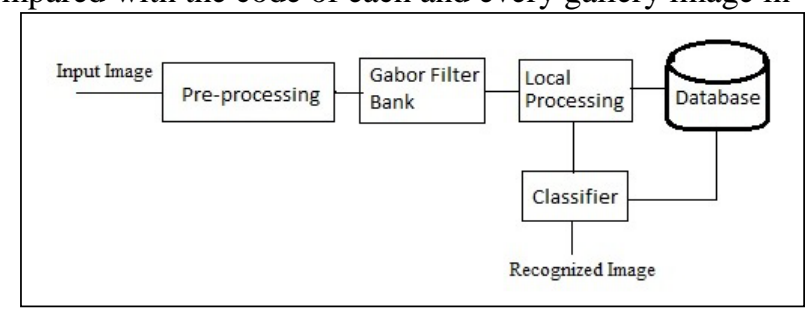

Fig. 1. Block diagram of Proposed System

the codebook to find out the correct match. The various steps performed in the proposed system are given in the Fig. 1.

\section{A. Preprocessing}

The input image is aligned, normalized and converted into $128 \times 128$ for each gallery and probe images. Histogram equalization method is used to improve the contrast of the image

\section{B. Feature Extraction}

Each input image is passed through a Gabor filter bank of size $\mathrm{MxN}$. Hence after filtering the input image we have number of Gabor filtered images with different scale and rotation. Each filtered image is then divided into PxQ regions. The histogram of each region is calculated and concatenated. Finally, all the concatenated histograms of a single input images are concatenated further to form face feature descriptor for that face image. With this process all the gallery images are processed and the database of feature descriptors for the gallery images is created.

\section{Filtering the image with Gabor filter bank:}

Gabor wavelets are proved to be effective in filtering the local spatial-frequency distribution, giving optimal resolution in both the domains.

The 2D Gabor wavelet in spatial domain and frequency domain is given by[16]

$$
\begin{aligned}
& \psi(\mathrm{x}, \mathrm{y})=\frac{f^{2}}{\pi \gamma \eta} \exp \left(-\left(\alpha^{2} \mathrm{x}_{\mathrm{r}}^{2}+\beta^{2} \mathrm{y}_{\mathrm{r}}^{2}\right)\right) \cdot \exp \left(\mathrm{j} 2 \pi \mathrm{f} \mathrm{x}_{\mathrm{r}}\right) \\
& \mathrm{x}_{\mathrm{r}}=\mathrm{x} \cdot \cos \theta+\mathrm{y} \cdot \sin \theta \& \mathrm{y}_{\mathrm{r}}=-\mathrm{x} \cdot \sin \theta+\mathrm{y} \cdot \cos \theta \\
& \Phi(\mathrm{u}, \mathrm{v})=e^{-\pi^{2}\left(\frac{1}{\alpha^{2}}\left(u_{r}-f\right)^{2}+\frac{1}{\beta^{2}} v_{r}^{2}\right)} \\
& \text { Where } \\
& \alpha=\frac{\sqrt{2} \pi f}{\sigma}, \beta=\frac{\sqrt{2} \pi f}{\sigma} \text { are the sharpness of Gaussian in } \mathrm{x}, \mathrm{y}
\end{aligned}
$$

direction respectively. ' $f$ ' is the central frequency of the sinusoidal function. $\sigma$ is the width of the Gaussian function representing standard deviation.

$\gamma=\frac{f}{\alpha}$ and $\eta=\frac{f}{\beta}$

$\theta$ is the orientation of the major axis of elliptical Gaussian.

To obtain the Gabor filtered image $g(x, y)$, the input image $f(x, y)$ is convolved with the Gabor filter. The output image is given as

$$
\mathrm{g}(\mathrm{x}, \mathrm{y})=\mathrm{f}(\mathrm{x}, \mathrm{y}) * \psi(\mathrm{x}, \mathrm{y})
$$

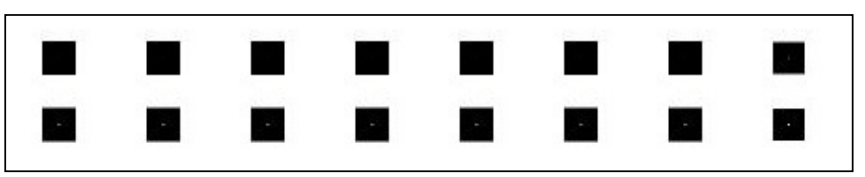

Fig. 2. Magnitude plot for the Gabor filter bank used

Where $(x, y)$ represents the co-ordinates and $f(x, y)$ represents the intensity of face image at that co-ordinate. Practically the convolution is determined by processing the functions in frequency domain. The Fourier Transform of input image and Gabor filter is determined and multiplied. To the obtained result inverse Fourier Transform is applied. This will represent a Gabor filtered image which is a complex function of $(\mathrm{u}, \mathrm{v})$, containing real and imaginary part. From which we can obtain easily its magnitude and phase. In our proposed system we are using only magnitude part.

\section{Local processing of the Gabor filtered images:}

The image $\mathrm{g}(\mathrm{x}, \mathrm{y})$ is divided into PxQ regions. The histogram of each region is calculated for the gray levels present using eqn. 4

$$
H_{(G)}^{p, q}=\sum_{(x, y) \epsilon R^{p, q}}(1) \text { for } \mathrm{G}=0,1, \ldots 255
$$

Where $G$ is the gray level. In multiresolution representation, the number of gray levels used may vary, i.e., 0 to 63,0 to 15 and 0 to 3 etc. $R^{p, q}$ represents the face region.

The histogram so obtained for each region are concatenated using eqn. 5 ,

$$
\mathrm{TH}=\prod_{i=1}^{N} H^{p, q}
$$

where $\Pi$ is concatenation operator and $\mathrm{N}=\mathrm{PXQ}$ is the total number of regions.

The operation is performed on all Gabor filtered images of single subject and finally they are concatenated using eqn. 6. This will give final face feature descriptor (FFD).

$$
\mathrm{FFD}=\prod_{i=1}^{N} G H^{i}
$$

where $\mathrm{N}$ is the number of Gabor filtered images. $\mathrm{GH}$ represents the complete histogram descriptor.

The feature vectors obtained in this way are then stored in the database as code book. 


\section{Classification: -}

In classification stage, an input probe image is processed in the same fashion as that of gallery images and the code is compared with the feature descriptors of gallery images stored in the code database. The closest match will be considered as recognized image. To find out this closest match we used Chi square similarity measure.

$$
\chi^{2}(\mathrm{~F} 1, \mathrm{~F} 2)=\sum \frac{[F 1-F 2(i)]^{2}}{F 1+F 2(i)}
$$

Where, F1 represents probe image and F2(i) represents gallery images.

\section{EXPERIMENTS AND RESULTS}

The input image is first aligned and normalized to size $128 \times 128$. The image is then passed through Gabor filter bank with 2 scales and 8 orientations. Due to this the dimensionality of the image gets multiplied by 2x8 times, i.e., the feature length is increased from $128 \times 128$ to $128 \times 128 \times 2 \times 8$. To reduce the dimensionality, we used down sampling of the Gabor filtered images with a down sampling factor of 16 . Hence for further processing, the feature length is $32 \mathrm{x} 32 \mathrm{x} 16$. This is for a single subject filtered with $2 \mathrm{x} 8$ filters. Each image of size $32 \times 32$ is thendivided into $4 \times 4$ regions and the histogram of each region is calculated separately. If we consider each gray level as a bin, the total number of bins will be 256, the length of the feature vector becomes 256x16x16 (number of bins x regions $x$ Gabor filtered images) $=65,536$. The histogram so obtained has very few nonzero elements, which will create redundancy in calculation. Therefore the number of bins are reduced further.

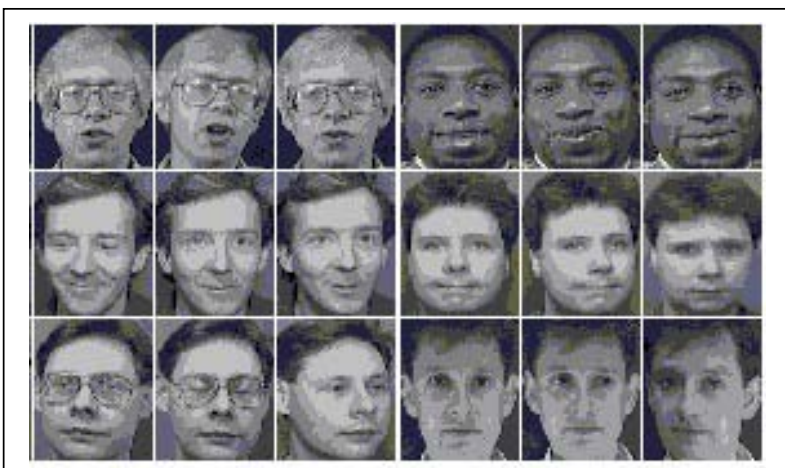

(a)

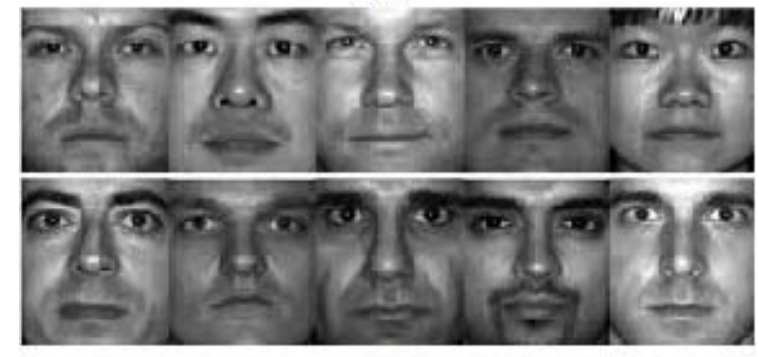

(b)

Fig. 3. Some of the faces in (a) AT\&T, (b) extended Yale B database

The length of feature vector can be reduced from 65536 to 16384 if number of bins are 64 , to 4096 if number of bins are $16 \&$ to 1024 if number of bins are 4 . Considering each case experiments are performed. The performance of the proposed system is evaluated using the publicly available AT\&T and extended YaleB databases.

\section{A. AT \& T Database}

The database contains 10 images of 40 subjects. The images are with different pose and expression conditions. The original image is of size $112 x 92$. In our experiment we resized it to $128 \times 128$ so as to have the equal sections during the division of the face images in local approach. Out of 10 face images per subject in the AT\&T database, the first three are taken as gallery and the last 4 images as a test images. Fig. 3(a) shows some of the faces with different pose and expression in AT\&T database[17].

Fig. 4 shows the feature vector with down sampling factor of 16 and 16 bins used for two different subjects. The marker shown at $500 \& 2500$ to show the variations in the feature values. The feature vector with different downsamling factor $1,4,16 \& 64$ is shown in the first column of Fig. 5 and the second column shows the featue vector with different number of bins 256, 64, 16 and 4 .

Number of experiments performed to find the optimum number of regions, to divide the face image for local processing from $1 \times 1=1$ region to $8 \times 8=64$ regions. The graph is showing increment in the recognition rate, as the number of regions are increasing. At $4 \times 4$ region we are getting maximum recognition rate for different number of bins considered as shown in Fig. 6(a), but after that the recognition rate is decresing for $8 \times 8$ regions.

In another expt, keeping down sampling factor constant, we vary the region size and noted the recognition rate for each region. The procedure is repeated for all four downsampling factor. The plot is shown in Fig. 6(b).

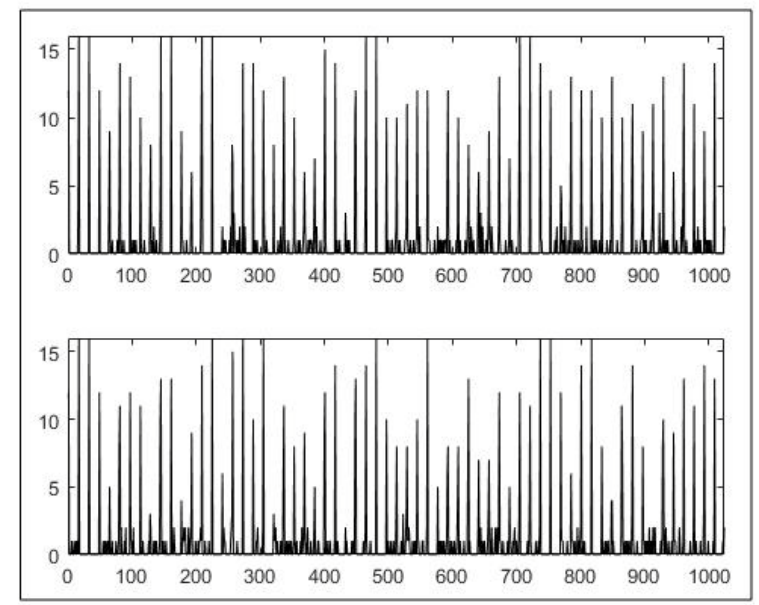

Fig. 4. Feature vector for two different subjects 


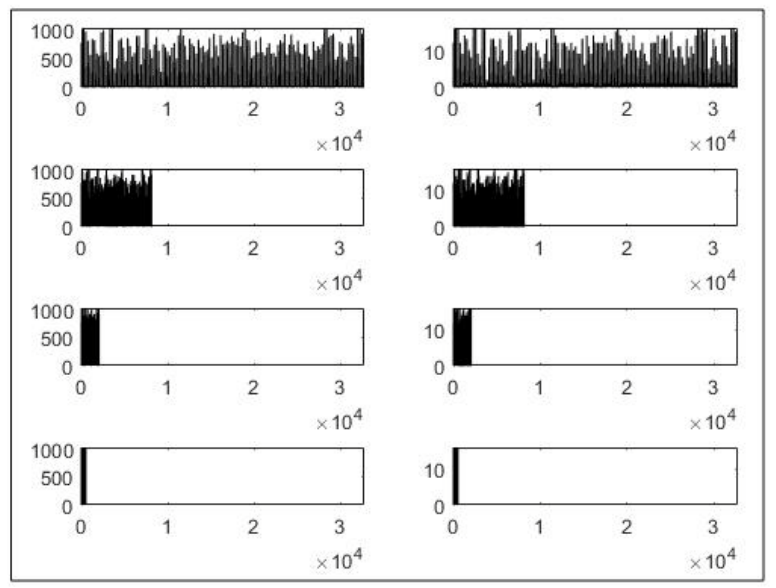

Fig. 5. Feature vector with diff. no. of bins \& down sampling factor

For down sampling $1 \& 4$ the results are maximum, but the computational cost and the time needed is also more.The result obtained by our proposed approach are compared with other such techniques. Table 1 gives the recognition rates obtained by different methods

Table I. Comparison of Different Methods (AT \& T Database)

\begin{tabular}{|c|c|c|}
\hline Sr. No. & Method used & $\begin{array}{c}\text { Recognition } \\
\text { Rate (\%) }\end{array}$ \\
\hline $\mathbf{1}$ & LBP & 83.75 \\
\hline $\mathbf{2}$ & LDN & 85.83 \\
\hline $\mathbf{3}$ & Gabor-PCA & 74.17 \\
\hline $\mathbf{4}$ & Gabor-LH & $\mathbf{9 2 . 1 2}$ \\
\hline
\end{tabular}

\section{B. Extended Yale B Database}

This database is mainly used for illumination variation evaluation. It contains images of 38 subjects, both with nine poses and 64 illuminations per pose[18]. From the available faces 10 faces per subject are taken. From the ten, first 3 are taken as gallery and last for as probe data, the remaining 3 are kept for the evaluation purpose. The comparison of the result is made with the result obtained by other method \& is tabulated in Table 2 .
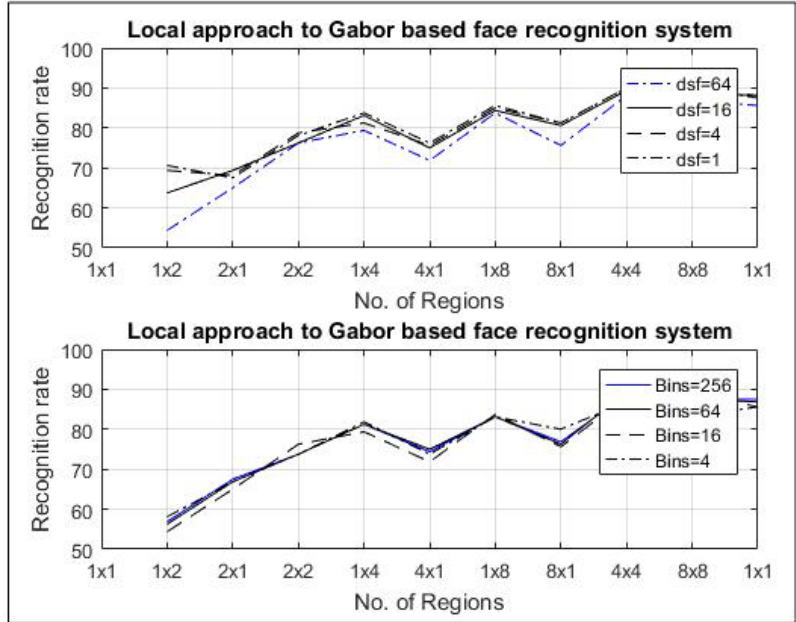

Fig. 6. Effect of varying number of regions on Recognition Rate

For different no.of bins, (b) diff. down sampling factor
Table II. Comparison of Different Methods (Extended YaleB Database)

\begin{tabular}{|c|c|c|}
\hline Sr. No. & Method used & $\begin{array}{c}\text { Recognition } \\
\text { Rate (\%) }\end{array}$ \\
\hline $\mathbf{1}$ & LBP & 84.16 \\
\hline $\mathbf{2}$ & LDN & 81.61 \\
\hline $\mathbf{3}$ & Gabor-PCA & 83.33 \\
\hline $\mathbf{4}$ & Gabor-LH & $\mathbf{9 4 . 1 5}$ \\
\hline
\end{tabular}

\section{CONCLUSIONS}

In this paper, we proposed a face recognition system using local histogram based approach. An input face image is filtered with Gabor filter bank to get the featured images. For creating the feature vector, the histogram of the various regions of these filtered images are determined separately and finally combined together. From the various experiments performed it can be concluded that -

- the region wise histogram is in the form of a sparse matrix and has very few nonzero elements.

- reducing the number of bins used in each region, equally preserves the results.

- $\quad$ thegabor filtered features uptodownsampling factor 64 can be effectively used for the classification of the face image.

This technique can be extended further, and only the selected feature descriptor with more discrimination can be used. Due to this added feature selection step, the computational time will be reduce.

\section{REFERENCES}

[1] P. Sinha, B. Balas, Y. Ostrovsky, R. Russell, Face Recognition by Humans: 19 Results All Computer Vision Researchers Should Know About, Proceedings of the IEEE, Vol. 94, No. 11, November 2006, pp. 1948-1962

[2] Linlin Shen \& Li Bai, "A review on Gabor wavelets for Face Recognition”, Pattern Analysis Application, vol. 9, 2006, pp. 273-292

[3] W. Zhao, R. Chellappa, A. Rosenfeld, P.J. Phillips, Face Recognition: A Literature Survey, ACM Computing Surveys, 2003, pp. 399-458

[4] Turk, M.A., Pentland, A.P., Eigenfaces for recognition. JournaL of Cognitive Neuroscience 3, 1991, pp.71-86.

[5] Belhumeur, P.N., Hespanha, J.P., Kriegman, D.J., Eigenfaces vs. fisherfaces: Recognitionusingclassspecificlinearprojection. IEEE Transactions on pattern analysis and machine intelligence 19, 1997, 711-720.

[6] Bartlett, M.S., Movellan, J.R., Sejnowski, T.J., 2002. Face recognition by independent component analysis. IEEE Transactions on neural networks 13, 1450-1464.

[7] Di Huang, Caifeng Shan, Mohsen Ardabilian, Yunhong Wang, and Liming Chen,"Local Binary Patterns and Its Application to Facial Image Analysis: A Survey", IEEE Trans.ON SYSTEMS, MAN, AND CYBERNETICS-PART C: APPLICATIONS AND REVIEWS, Vol. 41, No. 6, November 2011, pp. 765-781

[8] Xiaoyang Tan and Bill Triggs, "Enhanced Local Texture Feature Sets for Face Recognition Under Difficult Lighting Conditions", IEEE TRANSACTIONS ON IMAGE PROCESSING, VOL. 19, NO. 6, JUNE 2010, pp. 1635-1650

[9] Zhang, Y. Gao, S. Zhao, and J. Liu, "Local Derivative Pattern Versus Local Binary Pattern: Face Recognition With HighOrder Local Pattern Descriptor", IEEE TRANSACTIONS ON IMAGE PROCESSING, VOL. 19, NO. 2, FEBRUARY 2010, pp.533-544 
[10] Adin Ramirez Rivera, Jorge Rojas Castillo, and Oksam Chae, "Local Directional Number Pattern for Face Analysis: Face and Expression Recognition”, IEEE Transaction on Image Processing, Vol. 22, No. 5, May 2013, pp. 1740-1752

[11] Kuo-Chin Fan and Tsung-Yung Hung, "A Novel Local Pattern Descriptor-Local Vector Pattern in High-Order Derivative Space for Face Recognition”, IEEE Transactions on Image Processing, Vol. 23, No. 7, July 2014 pp. 2877-2891

[12] Lades, M., Distortion invariant object recognition in the dynamic link architecture. IEEE Trans on Computers 42, 1997, pp. 300-311

[13] Wiskott, J.-M., Fellous, N. Kruger, C.D. Von Malsburg, Face Recognition by Elastic Bunch Graph Matching, IEEE Transactions on Pattern Analysis and Machine Intelligence, Vol. 19, No. 7, July 1997, pp. 775-779

[14] Jie Zou, Qiang Ji, and George Nagy, "A Comparative Study of Local Matching Approach for Face Recognition", IEEE
TRANSACTIONS ON IMAGE PROCESSING, VOL. 16, NO. 10, OCTOBER 2007,pp. 2617-2628

[15] J. Ruiz-del-Solar, P. Navarrete, Eigenspace-based face recognition: a comparative study of different approaches, IEEE Transactions on Systems, Man and Cybernetics, Part C, Vol. 35, Issue 3, August 2005, pp. 315-325

[16] Kyrki, V., Kamarainen, J.K., Kalviainen, H., Simple Gabor feature space for invariant object recognition, Pattern Recognition Letters, 25, 2004, pp. 311-318

[17] Ferdinando Samaria, Andy Harter," Parameterisation of a Stochastic Model for Human Face Identification", Proceedings of 2nd IEEE Workshop on Applications of Computer Vision, Sarasota FL, December 1994

[18] The Extended Yale B database, http://vision.ucsd.edu/ iskwak/ExtYaleDatabase/ExtYaleB.ht $\mathrm{ml}$ 